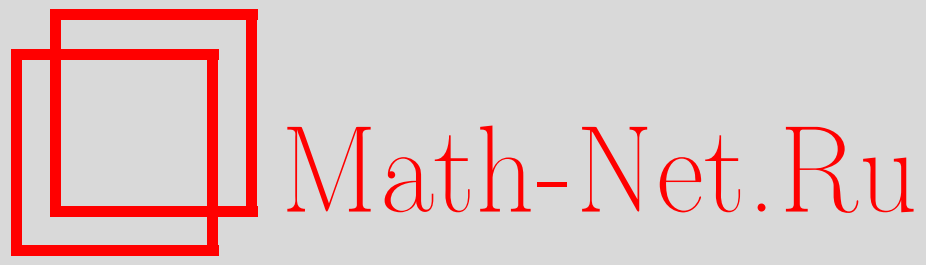

Р. Халер-Динтельман, В. Хёпнер, Г.-К. Кайзер, И. Реберг, Г. М. Циглер, Оптимальная эллиптическая регулярность в пространствах Соболева вблизи трехмерных многоматериальных вершин Неймана, Функи. анализ и его прил., 2014, том 48, выпуск $3,63-83$

DOI: https://doi.org/10.4213/faa3152

Использование Общероссийского математического портала MathNet.Ru подразумевает, что вы прочитали и согласны с пользовательским соглашением http://www . mathnet.ru/rus/agreement

Параметры загрузки:

IP : 35.173 .137 .237

26 апреля 2023 г., 09:56:39

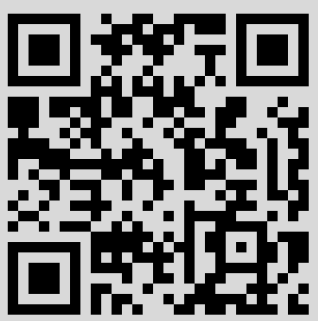




\title{
Оптимальная эллиптическая регулярность в пространствах Соболева вблизи трехмерных многоматериальных вершин Неймана*
}

\author{
(c) 2014. Р. ХАЛЕР-ДинтеЛЬмАН, В. ХЁПнеР, Х.-К. КАЙЗЕР,
} И. РЕБЕРГ, Г. М. ЦИГЛЕР

\begin{abstract}
Изучается оптимальная эллиптическая регулярность (в шкале пространств Соболева) анизотропных операторов дивергенции-градиента в размерности три вблизи многоматериальной вершины на неймановской части границы полиэдральной пространственной области. Градиент решения соответствующего эллиптического уравнения в частных производных в окрестности вершины интегрируем со степенью выше трех.
\end{abstract}

\section{$\S 1$. Введение}

В последние годы был установлен ряд результатов об эллиптической регулярности в шкале $W^{1, q}$ : в [12] и [30] - для оператора Лапласа с граничными условиями Дирихле или Неймана в областях с липшицевой границей, в [6] - для задачи Неймана и смешанных задач в полиэдрах, а в [3]-[5], [7], [9], [16], [21], [24], [25] - для случая разрывных коэффициентов; по поводу близких задач см. также [19], [20], [22]. В целом рассмотрен весьма любопытный набор геометрий и коэффициентов, включая даже случай смешанных граничных условий. Что не рассматривалось, так это оптимальная регулярность в пространствах $W^{1, p} \leftrightarrow W^{-1, p}$ в окрестности неймановской вершины в том случае, когда речь к тому же идет о разнородных материалах.

Интерес к таким задачам происходит в основном из естественных наук и техники, где многие явления описываются эллиптическими или параболическими уравнениями и часто важно учесть влияние разнородных материалов, которое математически выражается в виде разрывных функций в коэффициентах. Подробный список таких задач можно найти во введении к [9], а также в [4]. В частности, при анализе весьма широкого круга нелинейных задач (см. [10]) существенно, что пространство $W^{1, p}$ обладает «хорошими» мультипликаторными свойствами при условии, что $p$ больше, чем размерность пространства. Кроме того, читатель может представить себе параболическое уравнение с квадратичным градиентным членом в правой части. Тогда важно, с одной стороны, чтобы имелось вложение $L^{p / 2} \hookrightarrow W^{-1, p}$ (откуда в силу теоремы вложения Соболева следует, что $p$ должно быть больше, чем размерность пространства), а с другой стороны - вложение области определения эллиптического оператора в $W^{1, p}$, см. [10], [11]. Напомним, что пространства типа $W^{-1, q}$ содержат рас-

*Исследования Г. М. Циглера поддержаны исследовательским центром Немецкого научно-исследовательского общества MATHEON и расширенным грантом ERC no. 247029SDModels. 
пределения, такие, как, например, поверхностные плотности, необходимые во многих приложениях, см. [29, гл. 1].

Наш основной результат состоит, грубо говоря, в том, что существует $p>3$, такое, что для любой функции $f \in\left(W^{1, p^{\prime}}(\Pi)\right)^{\prime}$ любое решение $v$ уравнения $-\nabla \cdot \mu \nabla v=f$ лежит в $W^{1, p}$ локально вблизи любой вершины аء области П при условии, что $\bar{\Pi}$ - полиэдральное многообразие с краем, а коэффициентная функция $\mu$ обладает некоторыми свойствами, позволяющими оценивать соответствующие эллиптические реберные особенности (см. дальнейшие подробности в теореме 5.1).

Подчеркнем, что матрицы, составляющие коэффициентную функцию $\mu$, могут быть не диагональными и, в частности, не кратными единице (см. [15, гл. IV и V]). Это диктуется приложениями. Более того, анизотропные коэффициенты неизбежно возникают при (локальных) деформациях области (см. предложение 3.1). Следует заметить, что в случае анизотропной матрицы коэффициентов $\mu$ общие свойства эллиптического оператора $\nabla \cdot \mu \nabla$ могут разительно отличаться от таковых в случае скалярного коэффициента; см. [7, замечание 5.1], [8, §4] и [27, гл. 5].

Мы решаем задачу с помощью локализации, деформации и рассуждения, основанного на отражении. В частности, мы должны выпрямить часть границы таким образом, чтобы она стала частью плоскости. При этом, чтобы сохранить клеточную структуру областей постоянства преобразованной коэффициентной функции, необходимо в качестве преобразования использовать кусочно линейный гомеоморфизм. Поскольку наша цель - исследовать вершины общего вида, мы широко пользуемся нетривиальными (хотя и классическими) инструментами геометрической топологии в размерностях 2 и 3 . В качестве побочного результата мы получаем (утвердительный) ответ (теорема 3.10) на вопрос о том, каждое ли топологическое многообразие допускает липшицеву структуру (см. [17, гл. 9]), для случая трехмерных полиэдров, являющихся одновременно 3-многообразиями с краем.

\section{§ 2. Обозначения}

Всюду в тексте мы используем следующие обозначения. Через $\mathscr{C}:=]-1,1\left[^{3}\right.$ мы обозначаем открытый куб в $\mathbb{R}^{3}$ с центром в точке $0 ;$ кроме того, $\mathscr{C}_{ \pm}:=$ $\mathscr{C} \cap\left\{\mathrm{x}=(x, y, z) \in \mathbb{R}^{3}: y \gtrless 0\right\}$ и $\Sigma:=\mathscr{C} \cap\{\mathrm{x}=(x, 0, z): x, z \in \mathbb{R}\}$.

В том, что касается понятия липшицевой области, мы следуем терминологии из [18, разд. 1.1.9]. Пусть $\Omega-$ (ограниченная) липшицева область. Всюду далее через $\Gamma \subseteq \partial \Omega$ обозначается относительно открытая часть границы $\partial \Omega$. Символом $W^{1, p}(\Omega)$ обозначается обычное (комплексное) пространство Соболева на $\Omega$, а символом $W_{\Gamma}^{1, p}(\Omega)$ - замыкание множества

$$
\left\{\left.v\right|_{\Omega}: v \in C^{\infty}\left(\mathbb{R}^{3}\right), \operatorname{supp}(v) \cap(\partial \Omega \backslash \Gamma)=\varnothing\right\}
$$

в $W^{1, p}(\Omega)$. Если $\Gamma=\varnothing$, то, как обычно, мы пишем $W_{0}^{1, p}(\Omega)$ вместо $W_{\varnothing}^{1, p}(\Omega)$. Наконец, через $W_{\Gamma}^{-1, p^{\prime}}(\Omega)$ обозначается пространство непрерывных антилинейных форм на $W_{\Gamma}^{1, p}(\Omega), 1 / p+1 / p^{\prime}=1$.

Выражение $\langle\cdot, \cdot\rangle_{X}$ всегда обозначает спаривание банахова пространства $X$ и его (анти)дуального пространства; в случае если $X=\mathbb{C}^{d}$, мы обычно пи- 
шем $\langle\cdot, \cdot\rangle$. Если $\omega-$ измеримая по Лебегу существенно ограниченная функция на $\Omega$ со значениями в множестве $(d \times d)$-матриц, то мы определяем оператор $-\nabla \cdot \omega \nabla: W_{\Gamma}^{1,2}(\Omega) \rightarrow W_{\Gamma}^{-1,2}(\Omega)$ формулой

$$
\langle-\nabla \cdot \omega \nabla v, w\rangle_{W_{\Gamma}^{1,2}}:=\int_{\Omega} \omega \nabla v \cdot \nabla \bar{w} \mathrm{dx}, \quad v, w \in W_{\Gamma}^{1,2}(\Omega) .
$$

\section{§3. Преобразование задачи}

Все приемы, используемые нами при преобразовании задачи, существенно опираются на тот факт, что билипшицевы преобразования не изменяют ее общей структуры. Поэтому приведем сначала важную лемму из [9], позволяющую преобразовывать эллиптические операторы в дивергентной форме с помощью билипшицевых отображений с сохранением оптимальной регулярности.

Предложение $3.1\left[9\right.$, предложение 16]. Пусть $\Omega \subseteq \mathbb{R}^{d}$ - ограниченная липшицева область, а Г-открытое подмножество ее гранищы. Предположим, что $\phi$-билипшицево отображение окрестности множества $\bar{\Omega}$ в $\mathbb{R}^{d}, u$ введем обозначения $\phi(\Omega)=\Omega_{\star} u \phi(\Gamma)=\Gamma_{\star}$. Тогда справедливы следующие утверждения.

(i) Для любого $p \in] 1, \infty[$ отображение ф порождает линейный топологический изоморфизм

$$
\Psi_{p}: W_{\Gamma_{\star}}^{1, p}\left(\Omega_{\star}\right) \rightarrow W_{\Gamma}^{1, p}(\Omega),
$$

задаваемый формулой $\left(\Psi_{p} f\right)(x)=f(\phi(x))=(f \circ \phi)(x)$.

(ii) Oператор $\Psi_{p^{\prime}}^{*}$ является линейным топологическим изоморфизмом меж$\partial y W_{\Gamma}^{-1, p}(\Omega) u W_{\Gamma_{\star}}^{-1, p}\left(\Omega_{\star}\right)$.

(iii) Если $\omega$-ограниченная измеримая функция на $\Omega$ со значениями в множсестве $d \times d$-матрии, то

$$
\Psi_{p^{\prime}}^{*} \nabla \cdot \omega \nabla \Psi_{p}=\nabla \cdot \underline{\omega} \nabla
$$

əəe

$$
\underline{\omega}(\mathrm{y})=(D \phi)\left(\phi^{-1}(\mathrm{y})\right) \omega\left(\phi^{-1}(\mathrm{y})\right)(D \phi)^{T}\left(\phi^{-1}(\mathrm{y})\right) \frac{1}{\left|\operatorname{det}(D \phi)\left(\phi^{-1} \mathrm{y}\right)\right|},
$$

Dф - матрица Якоби отображения $\phi, a \operatorname{det}(D \phi)$ - ее определитель.

Кроме того, если отображение $-\nabla \cdot \omega \nabla: W_{\Gamma}^{1, p}(\Omega) \rightarrow W_{\Gamma}^{-1, p}(\Omega)-$ топологический изоморфизм, то топологическим изоморбизмом является и отображение $-\nabla \cdot \underline{\omega} \nabla: W_{\Gamma_{\star}}^{1, p}\left(\Omega_{\star}\right) \rightarrow W_{\Gamma_{\star}}^{-1, p}\left(\Omega_{\star}\right)$ (причем верно и обратное $)$.

\section{1. Локальное выпрямление границы кусочно линейными отобра-} жениями. В этом разделе мы докажем при весьма общих предположениях, что границу полиэдра в $\mathbb{R}^{3}$ можно локально (в окрестности любой ее точки) выпрямить кусочно линейным гомеоморфизмом. В частности, это означает, что если полиэдр разбит на клетки, в каждой из которых коэффициентная функция эллиптического оператора постоянна, то существует отображение, которое локально выпрямляет границу, не разрушая такую конфигурацию.

Для этого нам понадобятся некоторые понятия и результаты геометрической топологии, которые мы сейчас кратко и введем в духе книг Мойзе [23] и Александрова-Хопфа [1]; см. также Бинг [2]. 
Если $\mathrm{v}_{0}, \ldots, \mathrm{v}_{m} \in \mathbb{R}^{3}$, причем выпуклая оболочка этих точек содержит $d$-мерный шар и ни одного $(d+1)$-мерного шара, то эта выпуклая оболочка называется $d$-клеткой, порожденной точками $\mathrm{v}_{0}, \ldots, \mathrm{v}_{m}$. Стороны, ребра и вершины границы $d$-клетки называются гранями. Если $m \leqslant 3$ и точки $\mathrm{v}_{0}, \ldots, \mathrm{v}_{m} \in \mathbb{R}^{3}$ находятся в общем положении, то мы называем клетку симплексом. Симплексы в $\mathbb{R}^{3}$ суть тетраэдры, треугольники, ребра или вершины.

Евклидовым комплексом $K$ называется локально конечное семейство клеток в $\mathbb{R}^{3}$, такое, что $K$ содержит все грани всех своих элементов, причем если $\sigma$ и $\tau$-две клетки из $K$, такие, что $\sigma \cap \tau \neq \varnothing$, то $\sigma \cap \tau$ является гранью как клетки $\sigma$, так и клетки $\tau$ (см. [1, гл. III]). Комплекс называется симплициальным, если все его клетки - симплексы. Пусть $K$-комплекс в $\mathbb{R}^{3}$; тогда через $|K|:=\bigcup_{\sigma \in K} \sigma \subseteq \mathbb{R}^{3}$ обозначается полиэдр, задаваемый комплексом $K$. Если v - вершина евклидова комплекса $K$, то множество всех клеток из $K$, содержащих v, вместе со всеми их гранями образует звезду $K_{\mathrm{v}}^{\star}$ вершины v в $K$. Все те грани в этой звезде, для которых v не является вершиной, образуют линк вершины v. Открытая звезда определяется как теоретико-множественная разность звезды и линка. (Открытая звезда является открытым множеством в $|K|$ и не отвечает никакому подкомплексу.)

Если $K$-конечный симплициальный комплекс, такой, что $|K|$ является $m$-мерным топологическим многообразием (с краем), то комплекс $K$ называется триангулированным $m$-многообразием (с краем), а топологическое пространство $|K|$ - полиэдральным $m$-многообразием (с краем). Через $K_{\partial}$ мы обозначаем его граничный комплекс, порожденный всеми теми $(m-1)$-клетками исходного комплекса, каждая из которых лежит в точности в одной $m$-клетке. Все рассматриваемые в дальнейшем многообразия $M$ предполагаются компактными.

Если $K$ и $K^{\prime}$ - евклидовы комплексы в $\mathbb{R}^{3}$, такие, что $|K|=\left|K^{\prime}\right|$ и любая клетка $\sigma \in K^{\prime}$ содержится в некотором элементе из $K$, то $K^{\prime}$ называется подразделением комплекса $K$. Ниже нам неоднократно потребуется следующая важная связь между евклидовыми комплексами и симплициальными комплексами.

Предложение 3.2 [1, гл. III.2]. Каждый евклидов комплекс $K$ допускает симплициальное подразделение $K^{\prime}$ без новых вершин. Более того, для произвольной заранее заданной вершины $\mathrm{v} \in K$ подразделение $K^{\prime}$ можно построчть таким образом, что вериина $\mathrm{v}$ будет одним из концов каждого из ребер в $K^{\prime}$, которые окажутся в ее открытой звезде.

Пусть $K$ - комплекс в $\mathbb{R}^{d}$. Непрерывное отображение $f$ полиэдра $|K|$ на подмножество в $\mathbb{R}^{m}$ называется кусочно линейным, если существует подразделение $K^{\prime}$ комплекса $K$, такое, что сужение $\left.f\right|_{\sigma}-$ линейная функция для каждого $\sigma \in K^{\prime}$.

Замечание 3.3. В силу предложения 3.2 всегда можно предполагать, что клетки, на которых кусочно линейное отображение линейно, суть симплексы.

Если $K$ - конечный комплекс, а $f$ инъективно, то на $f(|K|)$ возникает структура комплекса, индуцированная из $K$ с помощью $f$. В случае симплициальных комплексов это определение совпадает с данным в [23]; ср. [2, гл. II]. В [23, гл. 5] можно также найти базовые сведения о кусочно линейных гомеоморфизмах. 
Наконец, нам понадобятся различные понятия края (границы) для многообразий и комплексов. Пусть $M$ есть $m$-мерное топологическое многообразие в $\mathbb{R}^{3}$, с краем или без. Тогда все точки из $M$, у которых есть открытая окрестность в $M$, гомеоморфная $\mathbb{R}^{m}$, образуют внутренность $\operatorname{Int}(M)$ многообразия $M$, а оставшееся множество $\operatorname{Bd}(M):=M \backslash \operatorname{Int}(M)$ называется теоретикомножественным краем многообразия $M$.

Символом $\partial A$ обозначается топологическая граница множества $A \subseteq \mathbb{R}^{d}$, т. е. $\partial A=\bar{A} \cap \overline{\mathbb{R}^{d} \backslash A}$, где замыкание следует брать в $\mathbb{R}^{d}$.

Замечание 3.4. Пусть $K$-триангулированное 3 -многообразие с краем. Предположим, что $\mu$ - коэффициентная функция на $|K|$, постоянная на внутренности каждой 3 -клетки из $K$, и что $\phi:|K| \rightarrow \mathbb{R}^{3}$ - кусочно линейное отображение, осуществляющее гомеоморфизм множества $|K|$ на его образ. Тогда полученная в результате коэффициентная функция на $\phi(|K|)$ (см. (3.1)) постоянна на внутренности каждой из 3-клеток, прообразы которых содержатся в 3-клетках из $K$.

В дальнейшем мы используем следующие результаты геометрической топологии.

Предложение 3.5 [2, теорема I.2.A]. Если $K$ - конечный симплициальный комплекс в $\mathbb{R}^{3}$, то существует триангуляиия $K_{\mathbb{R}^{3}}$ пространства $\mathbb{R}^{3}$, содержащая $K$ в качестве подкомплекса.

Предложение 3.6 ([17, теорема 2.18], [28, с. 504]). Пусть $K$ - конечный евклидов комплекс в $\mathbb{R}^{d}$, и пусть отображение $\phi:|K| \rightarrow \mathbb{R}^{m}$ кусочно линейно и непрерывно. Тогда оно непрерывно по Липшицу.

Предложение 3.7 (теорема Шёнфлиса для трехмерного кусочно линейного случая ([23, теорема 17.12], [2, теорема XIV.I])). Пусть $S-$ полиэдр в $\mathbb{R}^{3}$, гранииа которого топологически является 2-сферой. Тогда существует кусочно линейный гомеоморфизм $\phi_{S}: \mathbb{R}^{3} \rightarrow \mathbb{R}^{3}$, отображающий внутренность полиэдра $S$ на внутренность тетраэдра $\sigma^{3}$, а $\partial S-$ на $\partial \sigma^{3}$.

Замечание 3.8. Ниже мы применим теорему Шёнфлиса для трехмерного кусочно линейного случая, чтобы получить гомеоморфизм на границу не тетраэдра, а кирпича $\overline{\mathscr{C}}_{+}=[-1,1] \times[0,1] \times[-1,1]=\left\{(x, y, z) \in[-1,1]^{3}: y \geqslant 0\right\}$. Более того, нам потребуется, чтобы заданная точка а $\in S$ отображалась в точку $0 \in \partial \mathscr{C}_{+}$. Оба эти условия можно выполнить, построив кусочно линейный гомеоморфизм $\mathbb{R}^{3} \rightarrow \mathbb{R}^{3}$, отображающий $\sigma^{3}$ на $\overline{\mathscr{C}}_{+}$и переносящий заданную точку $\mathrm{a} \in \partial \sigma^{3}$ в 0 . Этого легко добиться явной геометрической конструкцией, в которой следует различать три случая, когда а лежит в относительной внутренности треугольной грани, на ребре или в вершине тетраэдра $\sigma^{3}$.

Докажем теперь ключевую лемму, которая понадобится для теоремы о выпрямлении в следующем разделе.

Лемма 3.9. Пусть $K$ - триангулированное 3 -многообразие с краем в $\mathbb{R}^{3}$, u пусть $\mathrm{v}$ - вершина на границе полиэдра $|K|$. Тогда полиэдр $\left|K_{\mathrm{v}}^{\star}\right|$ звезды вершины у в $K$ гомеоморфен замкнутому единичному шару в $\mathbb{R}^{3}$. B частности, краем многообразия $K_{\mathrm{v}}^{\star}$ является полиэдральная 2-сфера. 
Доказательство. Так как $|K|$ - трехмерное многообразие с краем и одновременно замкнутое подмножество в $\mathbb{R}^{3}$, то $\mathrm{Bd}|K|=\partial|K|$ (см. [23, теорема 17.1]). Далее, согласно [23, теорема 23.3], край $\mathrm{Bd}|K|$ есть 2-многообразие, которое также является полиэдром, отвечающим комплексу $K_{\partial}$, т.е. $\left|K_{\partial}\right|=$ $\mathrm{Bd}|K|$ (см. [23, теорема 23.7]). Следовательно, звезда $K_{\partial, \mathrm{v}}^{\star}$ вершины v $\in K_{\partial}$ представляет собой диск (см. [23, теорема 4.8]). Рассмотрим настолько малый шар $B$ с центром в $\mathrm{v}$, что он пересекает только те грани комплекса $K$, которые лежат в $K_{\mathrm{v}}^{\star}$, т. е. содержат v. Прежде всего, заметим, что $K_{\partial, \mathrm{v}}^{\star} \cap B$ гомеоморфно $K_{\partial, \mathrm{v}}^{\star}$. Поэтому пересечение диска $K_{\partial, \mathrm{v}}^{\star}$ с границей шара $B$ представляет собой жорданову кривую на 2-сфере $\partial B$, которая по двумерной теореме Шёнфлиса [23, теорема 10.2] делит сферу на два диска, одним из которых является $\partial B \cap K_{\mathrm{v}}^{\star}$. Таким образом, радиальная проекция из $\mathrm{v}$ показывает, что звезда $K_{\mathrm{v}}^{\star}$ является конусом (с вершиной v) над диском, а следовательно, шаром; его граница, будучи объединением двух дисков, а именно, звезды вершины v в $K_{\partial, \mathrm{v}}^{\star}$ и линка вершины v в $K_{\mathrm{v}}^{\star}$, является 2-сферой.

3.2. Теорема о кусочно линейном выпрямлении. Цель данного раздела состоит в том, чтобы установить следующий результат.

Теорема 3.10. Пусть $\Pi \subseteq \mathbb{R}^{3}$-область, такая, что $\bar{\Pi}$ - полиэдральное 3-многообразие с краем, удовлетворяющее условию $\Pi=\operatorname{Int}(\bar{\Pi})$. Тогда $\Pi-$ липшищева область и локальные билипшичевы карты вблизи точек ее границы могут быть заданы кусочно линейными гомеоморфизмами следующим образом.

Пусть $K_{\Pi}-$ евклидов комплекс, такой, что $\left|K_{\Pi}\right|=\bar{\Pi}$. Продолжим его до триангуляиии $K_{\mathbb{R}^{3}} \supset K_{\Pi}$ пространства $\mathbb{R}^{3}$. Тогда для любой точки а $\in \partial \Pi$ существует непрерывное кусочно линейное отображение $\varphi_{\mathrm{a}} к у б а \overline{\mathscr{C}}=[-1,1]^{3}$ $\subseteq \mathbb{R}^{3}$ на замкнутую окрестность $\mathscr{W}_{\text {а }}$ точки а в $\mathbb{R}^{3}$ со следующими свойствамu:

(1) точка 0 отображается в а;

(2) множество $\Sigma=]-1,1[\times\{0\} \times]-1,1[$ отображается в гранииу ӘП области П;

(3) множество $\left.\mathscr{C}_{+}=\right]-1,1[\times] 0,1[\times]-1,1[$ отображается в область $\Pi$;

(4) множество $\left.\mathscr{C}_{-}=\right]-1,1[\times]-1,0[\times]-1,1[$ отображается во внешность $\mathbb{R}^{3} \backslash \bar{\Pi}$ области П.

Кроме того, комплекс $L_{\overline{\mathscr{C}}}$, свлзанный с отображением $\varphi_{\mathrm{a}}$, обладает следующими свойствами:

(5) для любого $A \in L_{\overline{\mathscr{C}}}$ множество $\varphi_{\mathrm{a}}(A)$ является подмножеством элемента звезды вершины а в $K_{\mathbb{R}^{3}}$; в частности, если $A \in L_{\overline{\mathscr{C}}}-$ подмножество в $\overline{\mathscr{C}_{+}}$, то $\varphi_{\mathrm{a}}(A)-$ подмножество элемента звезды вериины а в $K_{\Pi}$.

(6) у каждой 3 -клетки комплекса $L_{\overline{\mathscr{C}}}$ одна из вершин лежит в точке 0 ;

(7) каждое ребро комплекса $L_{\overline{\mathscr{C}}}$, пересекающееся $c \mathscr{C}$, соединяет точку 0 c

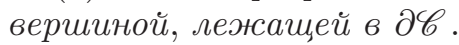

Замечание 3.11. Ключевые условия здесь - это равенство $\Pi=\operatorname{Int}(\bar{\Pi})$ и тот факт, что $\bar{\Pi}$ - многообразие с краем. Первое условие исключает трещины, а второе запрещает, например, призму с таким основанием, как показано на рис. 1, ср. замечание 4.3. 


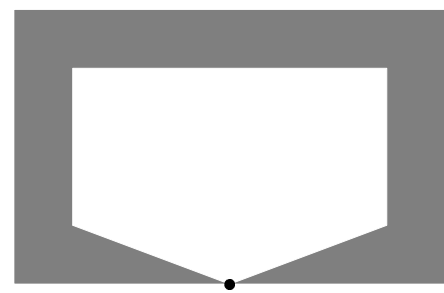

Рис. 1. Пинцет - не многообразие с краем, ср. замечание 3.11 .

Доказательство теоремы 3.10. Все утверждения теоремы легко установить для случая, когда а-относительно внутренняя точка граничного треугольника комплекса $K_{\Pi}$, и довольно несложно доказать в том случае, когда точка а лежит на ребре комплекса $K_{\Pi}$, поскольку тогда проекция параллельно этому ребру сводит дело к двумерной задаче. Трудным случаем является тот, в котором а - вершина комплекса $K_{\Pi}$.

Лемма 3.9 вместе с теоремой Шёнфлиса для трехмерного кусочно линейного случая, адаптированной согласно замечанию 3.8, дает кусочно линейный гомеоморфизм $h: \mathbb{R}^{3} \rightarrow \mathbb{R}^{3}$, отображающий звезду $K_{\text {a }}^{\star}$ на кирпич $\overline{\mathscr{C}_{+}}=[-1,1] \times$ $[0,1] \times[-1,1]$, а вершину а - в граничную точку $0 \in \partial \mathscr{C}_{+}$. Пусть $L_{\mathrm{ref}}-$ подразделение комплекса $K_{\mathbb{R}^{3}}$ и одновременно измельчение триангуляции пространства $\mathbb{R}^{3}$, связанной с кусочно линейным гомеоморфизмом $h: \mathbb{R}^{3} \rightarrow \mathbb{R}^{3}$ (с подкомплексом, триангулирующим $\left.K_{a}^{\star}\right)$. С точностью до еще одного подразделения (не сужающего полиэдр звезды вершины а), можно предположить, что 3 -клетки комплекса $L_{\mathrm{ref}}$ суть тетраэдры; мы перейдем к этому подразделению, но сохраним обозначение $L_{\text {ref }}$.

Пусть $L_{\text {a }}^{\star}$ - звезда вершины а в $L_{\text {ref }}$. Рассмотрим теперь комплекс $h\left(L_{a}^{\star}\right)$. Полиэдр $\left|h\left(L_{\mathrm{a}}^{\star}\right)\right|$ является окрестностью нуля. Очевидно, что все клетки комплекса $h\left(L_{\mathrm{a}}^{\star}\right)$ - снова тетраэдры, на этот раз с одной вершиной в нуле. Кроме того, все ребра, пересекающие внутренность полиэдра $\left|h\left(L_{\mathrm{a}}^{\star}\right)\right|$, выходят из нуля. Пусть $\bar{\varepsilon} \bar{C}-$ куб, лежащий во внутренности полиэдра $\left|h\left(L_{\mathrm{a}}^{\star}\right)\right|$. Таким образом, пересечение с $\left|h\left(L_{\mathrm{a}}^{\star}\right)\right|$ индуцирует на $\varepsilon \overline{\mathscr{C}}$ структуру комплекса, у клеток которого одна из вершин находится в нуле. Далее, отображение $\varphi_{\mathrm{a}}:=\left.h^{-1}\right|_{\varepsilon \overline{\mathscr{C}}}$ линейно на каждой из этих клеток. Итак, окончательно, масштабируя х $\mapsto \varepsilon x$ и полагая $\mathscr{W}_{\mathrm{a}}:=\varphi_{\mathrm{a}}(\overline{\mathscr{C}})$, получаем нужную окрестность. Необходимое свойство билипшицевости отображения $\varphi_{\text {a }}$ следует из предложения 3.6.

\section{§4. Реберные особенности}

В этом параграфе, после некоторых приготовлений, мы напомним результат об оптимальной регулярности, доказанный в [21] для гетерогенных задач Дирихле в полиэдральных областях, и объясним, как идентифицировать возникающие реберные особенности. Сначала введем некоторые понятия и обозначения, отвечающие нашей геометрической ситуации с полиэдральной областью П и кусочно постоянной коэффициентной функцией $\mu$.

Определение 4.1. Пусть $\iota, \vartheta \in]-\pi, \pi], \iota<\vartheta$. Определим сектор

$$
S_{\iota}^{\vartheta}:=\{(r \cos \theta, r \sin \theta): r>0, \theta \in] \iota, \vartheta[\} .
$$


Определение 4.2. Пусть $\Omega \subseteq \mathbb{R}^{3}$ - липшицева область, такая, что $\bar{\Omega}$ - полиэдральное 3 -многообразие с краем, ассоциированное с конечным комплексом $K$. Если $\left\{\sigma_{k}\right\}_{k}$ - множество всех 3 -клеток из $K$, то определим $\mu$ как матричную функцию на $\Omega$, постоянную на внутренности каждой клетки $\sigma_{k}$, значения которой - вещественные симметрические положительно определенные матрицы размера $3 \times 3$. Возьмем ребро $E$ любой из клеток $\sigma_{k}$ и рассмотрим произвольную точку $P$ этого ребра, не являющуюся его концом.

Выберем новую ортогональную систему координат $(x, y, z)$ с началом в точке $P$, такую, что направление ребра $E$ совпадает с осью $z$. Через $\mathscr{O}_{E}$ обозначим матрицу соответствующего ортогонального преобразования, а через $\mu_{E, P}-$ кусочно постоянную матричную функцию, совпадающую для х из окрестности точки $0 \in \mathbb{R}^{3}$ с $A_{E, P} \mathrm{x}:=\mathscr{O}_{E} \mu\left(\mathscr{O}_{E}^{-1} \mathrm{x}+P\right) \mathscr{O}_{E}^{-1}$ и удовлетворяющую условию

$$
\mu_{E, P}(t x, t y, z)=\mu_{E, P}(x, y, 0) \quad \text { для всех }(x, y, z) \in \mathbb{R}^{3}, t>0 .
$$

Через $\mu_{E}(\cdot, \cdot)$ обозначим верхний левый $2 \times 2$-блок матрицы $\mu_{E, P}(\cdot, \cdot, 0)$.

Замечание 4.3. Существенно, что, как и указывают наши обозначения, коэффициентная функция $\mu_{E}$ - одна и та же для всех точек $P$ из (относительной) внутренности ребра $E$.

Существуют углы $\theta_{0}<\theta_{1}<\cdots<\theta_{n} \leqslant \theta_{0}+2 \pi$, такие, что $\mu_{E}$ постоянна в каждом из секторов $S_{\theta_{j}}^{\theta_{j+1}}$ и принимает значения в множестве вещественных симметрических положительно определенных матриц. В дальнейшем такие коэффициентные функции в секторе $S \subseteq \mathbb{R}^{2}$ будут называться посекторно постоянными.

Если $\mu_{E}$ отвечает внутреннему ребру $E$, то $\theta_{n}=\theta_{0}+2 \pi$. В противном случае $\mu_{E}$ задана на бесконечном секторе $S_{\theta_{0}}^{\theta_{n}}$, который вблизи $P$ совпадает с пересечением преобразованной области $\Omega$ с плоскостью $(x, y)$.

Заметим, что полный сектор $S_{\theta_{0}}^{\theta_{n}}$ в случае граничных ребер появляется в действительности благодаря тому факту, что $\bar{\Omega}$ - полиэдральное 3 -многообразие с краем.

Чтобы привести результат из [21] об оптимальной регулярности, введем теперь оператор Штурма-Лиувилля, ассоциированный с ребром и коэффициентной функцией $\mu$.

Определение 4.4. Пусть заданы числа $\theta_{0}<\theta_{1}<\cdots<\theta_{n} \leqslant \theta_{0}+2 \pi$, а также вещественные симметрические положительно определенные $2 \times 2$-матрицы $\rho^{1}, \ldots, \rho^{n}$, ассоциированные с секторами $S_{\theta_{0}}^{\theta_{1}}, \ldots, S_{\theta_{n-1}}^{\theta_{n}}$. Обозначим получающуюся коэффициентную функцию на всем секторе $S_{\theta_{0}}^{\theta_{n}}$ через $\rho$. Введем на множестве $] \theta_{0}, \theta_{n}\left[\backslash\left\{\theta_{1}, \ldots, \theta_{n-1}\right\}\right.$ коэффициентные функции $b_{0}, b_{1}, b_{2}$, сужения которых на интервал $] \theta_{j}, \theta_{j+1}[, j=0, \ldots, n-1$, заданы формулами

$$
\begin{aligned}
& b_{0}(\theta)=\rho_{11}^{j} \cos ^{2} \theta+2 \rho_{12}^{j} \sin \theta \cos \theta+\rho_{22}^{j} \sin ^{2} \theta, \\
& b_{1}(\theta)=\left(\rho_{22}^{j}-\rho_{11}^{j}\right) \sin \theta \cos \theta+\rho_{12}^{j}\left(\cos ^{2} \theta-\sin ^{2} \theta\right), \\
& b_{2}(\theta)=\rho_{11}^{j} \sin ^{2} \theta-2 \rho_{12}^{j} \sin \theta \cos \theta+\rho_{22}^{j} \cos ^{2} \theta .
\end{aligned}
$$

Если $\theta_{n} \neq \theta_{0}+2 \pi$, то определим пространство $\mathscr{H}$ как $W^{1,2}(] \theta_{0}, \theta_{n}[)$ в случае условия Неймана и как $W_{0}^{1,2}(] \theta_{0}, \theta_{n}[)$ в случае условия Дирихле. Если $\theta_{n}=$ 
$\theta_{0}+2 \pi$ (случай внутреннего ребра), то определим $\mathscr{H}$ как периодическое пространство Соболева $W^{1,2}(] \theta_{0}, \theta_{n}[) \cap\left\{v: v\left(\theta_{0}\right)=v\left(\theta_{n}\right)\right\}$, которое очевидным образом можно идентифицировать с пространством Соболева $W^{1,2}\left(S^{1}\right)$ на единичной окружности $S^{1}$. Для каждого $\lambda \in \mathbb{C}$ определим квадратичную форму $\mathfrak{t}_{\lambda}$ на $\mathscr{H}$, полагая

$$
\mathfrak{t}_{\lambda}[v]:=\int_{\theta_{0}}^{\theta_{n}}\left(b_{2} v^{\prime} \overline{v^{\prime}}+\lambda b_{1} v \overline{v^{\prime}}-\lambda b_{1} v^{\prime} \bar{v}-\lambda^{2} b_{0} v \bar{v}\right) \mathrm{d} \theta,
$$

и $\mathscr{A}_{\lambda}$ как оператор, индуцированный формой $\mathfrak{t}_{\lambda}$ на пространстве $L^{2}(] \theta_{0}, \theta_{n}[)$ или на $L^{2}\left(S^{1}\right)$ соответственно.

Как обычно [19], семейство $\left\{\mathscr{A}_{\lambda}\right\}_{\lambda}$ будет в дальнейшем называться соответствующим операторным пучком.

Определение 4.5. Пусть $E$ - произвольное ребро триангуляции множества $\bar{\Omega}$, и пусть $\mathscr{A}_{\lambda}$ задано, как в определении 4.4 , с $\rho=\mu_{E}$. Назовем число

$$
\inf \left\{\operatorname{Re} \lambda>0: \operatorname{ker}\left(\mathscr{A}_{\lambda}\right) \neq\{0\}\right\}
$$

показателем сингулярности, ассоциированным с $E$. Более общо, число $\lambda$, $\operatorname{Re} \lambda>0$, такое, что $\operatorname{ker}\left(\mathscr{A}_{\lambda}\right) \neq\{0\}$, будем называть сингулярным числом операторного пучка $\mathscr{A}_{\lambda}$.

Приведем теперь центральный результат о линейной регулярности из [21, теорема 2.3], который в дальнейшем послужит основным инструментом в доказательстве нашего результата о регулярности.

Предложение 4.6. Пусть $\Omega,\left\{\sigma_{k}\right\}_{k}$ u $\mu$ такие жее, как в определении 4.2. Если для любого ребра триангуляиии $\left\{\sigma_{k}\right\}_{k}$ ассоииированный показатель сингулярности больше, чем 1/3, то существует $p>3$, такое, что

$$
-\nabla \cdot \mu \nabla: W_{0}^{1, p}(\Omega) \rightarrow W^{-1, p}(\Omega)
$$

- топологический изоморфизм.

Замечание 4.7. Хорошо известно, что в любой полосе $\left\{\lambda=\lambda_{1}+i \lambda_{2}\right.$ : $\left.\left|\lambda_{1}\right| \leqslant c\right\}$ содержится лишь конечное число значений $\lambda$, для которых ядро оператора $\mathscr{A}_{\lambda}$ нетривиально, см. [21, следствие 3.11]. Поэтому достаточно показать, что ни у одного из операторов $\mathscr{A}_{\lambda}$ с $0<\operatorname{Re} \lambda \leqslant 1 / 3$ нет нетривиального ядра.

В последующих параграфах все ребра, возникающие в нашей задаче, изучаются на предмет тривиальности ядра $\operatorname{ker}\left(\mathscr{A}_{\lambda}\right)$. Здесь существенны два типа ребер, а именно, мономатериальные ребра и биматериальные внешние ребра, описываемые в следующем определении.

Определение 4.8. Пусть $E$ - ребро многообразия $\bar{\Omega}$, лежащее в $\partial \Omega$. Используя терминологию из определения 4.2, скажем, что

(i) $E$ - мономатериалъное ребро, если у каждой точки в относительной внутренности ребра $E$ имеется окрестность в $\bar{\Omega}$, на которой $\mu$ постоянна п. в. относительно трехмерной меры Лебега;

(ii) E-биматериальное внешнее ребро, если у функции $\left[\theta_{0}, \theta_{n}\right] \ni \theta \mapsto$ $\mu_{E}(\cos (\theta), \sin (\theta))$ есть ровно один скачок, причем длины обоих интервалов постоянства этой функции не превосходят $\pi$. Здесь $\theta_{0}$ и $\theta_{n}-$ углы, ассоциированные с $E$ в замечании 4.3. 
Для соответствующих особенностей имеются следующие два результата (см. [7, лемма 2.3] или [9, теорема 24/25]).

Предложение 4.9. Для любого мономатериального ребра Е ядра ассоциированньх с ним операторов $\mathscr{A}_{\lambda}$ тривиальны, если ставится чистое условие Дирихле или чистое условие Неймана, $a \operatorname{Re} \lambda \in] 0,1 / 2]$.

Предложение 4.10. Пусть $S_{\theta_{0}}^{\theta_{1}}, S_{\theta_{1}}^{\theta_{2}}$ - два соседних сектора в $\mathbb{R}^{2}$, такие, что $\theta_{1}-\theta_{0}, \theta_{2}-\theta_{1} \leqslant \pi$ и $\theta_{2}-\theta_{0}<2 \pi$. Пусть $\rho^{1}, \rho^{2}$ - вещественные симметрические положительно определенные $2 \times 2$-матричь, отвечающие секторам $S_{\theta_{0}}^{\theta_{1}}$ и $S_{\theta_{1}}^{\theta_{2}}$ соответственно. Пусть $\mathfrak{t}_{\lambda}-$ форма, определенная соотношением (4.2) на $W_{0}^{1,2}(] \theta_{0}, \theta_{2}[)$ или на $W^{1,2}(] \theta_{0}, \theta_{2}[)$. Тогда существует $\varepsilon>0$, такое, что ядро соответствующего оператора $\mathscr{A}_{\lambda}$ (см. определение 4.4) тривиально при $\operatorname{Re} \lambda \in] 0,1 / 2+\varepsilon]$.

В оставшейся части этого параграфа собраны некоторые необходимые в дальнейшем результаты о реберных особенностях.

Прежде всего установим принцип инвариантности для множества сингулярных чисел операторного пучка $\mathscr{A}_{\lambda}$ при действии посекторно линейного отображения на геометрическую и коэффициентную конфигурации.

Определение 4.11. Пусть $S \subseteq \mathbb{R}^{2}$ - сектор, который разбит на подсекторы $\widehat{S}_{1}, \ldots, \widehat{S}_{m}$, такие, что $\widehat{S}_{l}$ соседствует с $\widehat{S}_{l-1}$ и $\widehat{S}_{l+1}, l=2, \ldots, m-1$. Пусть $A-$ гомеоморфизм сектора $S$ на другой сектор $S_{\bullet}$. Будем говорить, что $A$ noceкторно линеен, если его ограничение на каждый сектор $\widehat{S}_{l}-$ линейное отображение, т. е. если из того, что $\mathrm{x}, \mathrm{y} \in \widehat{S}_{l}$ и $\mathrm{x}+$ у также лежит в $\widehat{S}_{l}$, вытекает, что $A \mathrm{x}+A \mathrm{y}=A(\mathrm{x}+\mathrm{y})$, и, кроме того, $A(t \mathrm{x})=t A \mathrm{x}$ при $\mathrm{x} \in \widehat{S}_{l}$ и $t>0$.

Замечание 4.12. Заметим, что отображение, обратное к посекторно линейному отображению, само посекторно линейно.

Располагая этими сведениями, можно доказать следующую теорему.

Теорема 4.13. Пусть задано разбиение сектора $S \subseteq \mathbb{R}^{2}$ на подсекторь

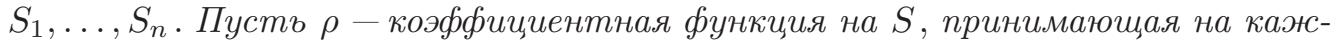
дом из секторов $S_{l}$ постоянное значение $\rho_{l}$, представляющее собой симметрическую положительно определенную матричу размера $2 \times 2$. Далее, пусть $A: S \rightarrow S_{\bullet}$ - посекторно линейный гомеоморфизм. Тогда справедливы следующие утверждения.

1. Преобразованная коэфбициентная функи, $\underline{\rho}$ (см. предложсение 3.1) также посекторно постоянна на $S_{\bullet}$.

2. Если $\mathscr{A}_{\lambda}$ - операторный пучок, ассочиированный с коэффициентной функиией $\rho$, а $\mathscr{A}_{\lambda}$ - соответствующий операторный пучок для коэффиииентной

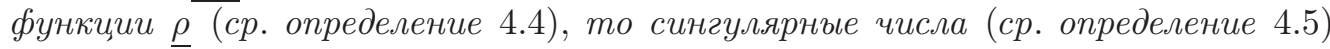
пучков 㔫 и $\mathscr{A}_{\lambda}$ в действительности совпадают. В частности, показатели сингулярности этих пучков одинаковы.

Доказательство. Первое утверждение следует из формулы преобразования (3.1). Второе утверждение доказано в [13, теорема 4.3] для случая, когда $\theta_{n} \neq \theta_{0}+2 \pi$ и сектор $S$ разбит только на два подсектора $\widehat{S}_{1}, \widehat{S}_{2}$. Достаточно очевидных изменений, чтобы перенести это доказательство на общий случай. Ключевым является тот факт, что посекторно линейное отображение однородно степени 1. 
Замечание 4.14. Заметим, что разбиения на секторы $S_{1}, \ldots, S_{n}$ и $\widehat{S}_{1}, \ldots, \widehat{S}_{m}$ совершенно друг от друга не зависят. Тем не менее они имеют общее подразбиение.

Теорема 4.15. Пусть $K_{E}$-евклидов комплекс в $\mathbb{R}^{3}$ и выполнены следующие предположения.

1. Каждая 3-клетка $\sigma \in K_{E}$ содержит ребро $E$.

2. Если $P \in E$ является произвольной (относительно) внутренней точкой, a $\mathscr{H}(P, E)$ - плоскость, содержащая $P$ и перпендикулярная $E$, то пересечение $\left|K_{E}\right| \cap \mathscr{H}(P, E)$ локально вблизи $P$ совпадает с полным сектором.

3. $\omega$ - коэффициентная функиия на $\left|K_{E}\right|$, принимающая значения в множестве вещественных симметричных положительно определенных $3 \times 3$-матрич, $u$ постоянная на каждой клетке $\sigma \in K_{E}$.

4. Отображсение $\phi:\left|K_{E}\right| \rightarrow \mathbb{R}^{3}$ является гомеоморфизмом множества $\left|K_{E}\right|$ на его образ и линейно на каэндой клетке $\sigma \in K_{E}$.

Тогда преобразованная коэффиииентная функиия (см. предложение 3.1)

$$
\rho(\mathrm{y})=(D \phi)\left(\phi^{-1}(\mathrm{y})\right) \omega\left(\phi^{-1}(\mathrm{y})\right)(D \phi)^{T}\left(\phi^{-1}(\mathrm{y})\right) \frac{1}{\left|\operatorname{det}(D \phi)\left(\phi^{-1} \mathrm{y}\right)\right|}
$$

на полиэдре $\phi\left(\left|K_{E}\right|\right)$ удовлетворяет (с необходимыми изменениями) предположсению 3, и, кроме того, сингулярные числа соответствующих операторных пучков $\mathscr{A}_{\lambda}$ и $\mathscr{A}_{\lambda}$ (ср. определение 4.4) одинаковы. В частности, совпадают их показатели сингулярности (ср. определение 4.5).

Доказательство. Мы покажем, что коэффициентные функции $\omega_{E}$ и $\rho_{\phi(E)}$ (ср. определение 4.2) связаны посекторно линейным отображением, что позволяет применить теорему 4.13.

Напомним, как получается коэффициентная функция $\omega_{E}$, определяющая операторный пучок $\mathscr{A}_{\lambda}$ : рассматривается аффинное преобразование $A_{E, P}$, переводящее произвольную точку $P \in E$ в начало координат и поворачивающее (сдвинутое) ребро $E$ таким образом, чтобы оно совпало с осью $z$, см. определение 4.2. Коэффициентная функция $\omega_{E, P}$ совпадает на достаточно малой окрестности $\mathscr{V}$ начала координат $0 \in \mathbb{R}^{3}$ с коэффициентной функцией, получающейся из $\omega$ в результате преобразования $A_{E, P}$, ср. предложение 3.1, и продолжается на все $\mathbb{R}^{3}$ по формуле (4.1). Наконец, $\omega_{E}$ - верхний левый блок матрицы $\omega_{E, P}(\cdot, \cdot, 0)$ и, таким образом, уже не зависит от $P$, ср. определение 4.2 .

Ту же конструкцию мы применяем к комплексу $\phi\left(K_{E}\right)$ и соответствующей коэффициентной функции $\rho$, определяя таким образом отображение $A_{\phi(E), \phi(P)}$ и операторный пучок $\frac{\mathscr{A}_{\lambda}}{}$. Отображение $B:=A_{\phi(E), \phi(P)} \phi A_{E, P}^{-1}: A_{E, P}\left|K_{E}\right| \rightarrow$ $A_{\phi(E), \phi(P)} \phi\left(\left|K_{E}\right|\right)$ - гомеоморфизм, действующий линейно на каждом клине $\left.S_{l} \times\right]-\infty, \infty\left[\right.$ где $S_{l}$ - любой из секторов, определенных в замечании 4.3 и отвечающих здесь коэффициентной функции $\omega$. Заметим, что $B$ отображает $0 \in \mathbb{R}^{3}$ в себя и переводит вектор $(0,0,1)^{T}$ в вектор $(0,0, \tau)^{T}$, где $\tau \neq 0$. Поэтому сужение отображения $B$ на любой из этих клиньев должно быть линейным отображением вида

$$
B_{l}=\left(\begin{array}{lll}
b_{11} & b_{12} & 0 \\
b_{21} & b_{22} & 0 \\
b_{31} & b_{32} & \tau
\end{array}\right)
$$


Величина $\tau$ не зависит от конкретного клина, но $|\tau|$ является коэффициентом растяжения ребра $E$ относительно $\phi$. В соответствии с формулой преобразования (3.1) для точек х из соответствующего клина-образа, которые к тому же близки к $A_{\phi(E), \phi(P)} \phi(E)$, имеем

$$
\rho_{\phi(E), \phi(P)}(\mathrm{x})=\frac{1}{\left|\operatorname{det}\left(B_{l}\right)\right|} B_{l} \omega_{E, P}\left(B_{l}^{-1}(\mathrm{x})\right) B_{l}^{T} .
$$

Однако ввиду (4.1) соотношение (4.3) распространяется на весь клин-образ. Для $3 \times 3$-матрицы $M$ пусть $\widehat{M}$ обозначает верхний левый блок размера $2 \times 2$. Матрица $\widehat{B}$ порождает посекторно линейное отображение соответствующего сектора, который является частью плоскости $(x, y)$. Поскольку $B$ имеет специальный вид, а именно, $b_{13}=b_{23}=0$, мы получаем соотношение

$$
\widehat{\rho}_{\phi(E), \phi(P)}(\mathrm{x})=\frac{1}{|\operatorname{det}(B)|} \widehat{B} \widehat{\omega}_{E, P}\left(B^{-1}(\mathrm{x})\right) \widehat{B}^{T}=\frac{1}{|\tau|} \frac{1}{|\operatorname{det}(\widehat{B})|} \widehat{B} \widehat{\omega}_{E, P}\left(B^{-1}(\mathrm{x})\right) \widehat{B}^{T}
$$

при $\mathrm{x} \in A_{\phi(E), \phi(P)} \phi\left(\left|K_{E}\right|\right)$. Отсюда следует, что

$$
\rho_{\phi(E)}(x, y)=\widehat{\rho}_{\phi(E), \phi(P)}(x, y, 0)=\frac{1}{|\tau|} \frac{1}{|\operatorname{det}(\widehat{B})|} \widehat{B}_{\widehat{\omega}_{E, P}}\left(B^{-1}(x, y, 0)\right) \widehat{B}^{T} .
$$

Далее, опять-таки в силу специального вида матрицы $B$, получаем

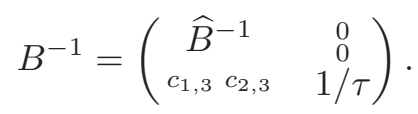

Подстановка этого выражения в (4.4) дает

$$
\rho_{\phi(E)}(x, y)=\frac{1}{|\tau|} \frac{1}{|\operatorname{det}(\widehat{B})|} \widehat{B} \widehat{\omega}_{E, P}\left(\widehat{B}^{-1}(x, y), z\right) \widehat{B}^{T}
$$

при $z=c_{1,3} x+c_{2,3} y \in \mathbb{R}$. Однако коэффициентные функции $\omega_{E, P}$ и $\widehat{\omega}_{E, P}$ не зависят от $z$, так что окончательно получаем

$\rho_{\phi(E)}(x, y)=\frac{1}{|\tau|} \frac{1}{|\operatorname{det}(\widehat{B})|} \widehat{B} \widehat{\omega}_{E, P}\left(\widehat{B}^{-1}(x, y), 0\right) \widehat{B}^{T}=\frac{1}{|\tau|} \frac{1}{|\operatorname{det}(\widehat{B})|} \widehat{B} \omega_{E}\left(\widehat{B}^{-1}(x, y)\right) \widehat{B}^{T}$.

Итак, коэффициентная функция $\rho_{\phi(E)}$ получается из коэффициентной функции $\omega_{E}$ посекторно линейным отображением (с точностью до постоянного скалярного множителя $\left.\frac{1}{|\tau|}\right)$ и наоборот. Поэтому в силу теоремы 4.13 ядро $\operatorname{ker} \mathscr{A}_{\lambda}$ тривиально при условии, что тривиально ядро ker $\mathscr{A}_{\lambda}$.

Последний результат этого параграфа в некотором смысле является обращением рассуждения, основанного на отражении (ср. леммы 6.3 и 6.4 ниже): он позволяет держать под контролем сингулярности на ребрах из $\Sigma$, получающиеся из четного отражения относительно бывшей неймановской части границы.

Лемма 4.16. Пусть полупространство $\{(x, y): y>0\}$ разбито на секторь $S_{0}^{\theta_{1}}, \ldots, S_{\theta_{n-1}}^{\pi}$ с ассочиированньми матрищами

$$
\left(\begin{array}{cc}
\rho_{11}^{1} & \rho_{12}^{1} \\
\rho_{12}^{1} & \rho_{22}^{1}
\end{array}\right), \quad \cdots, \quad\left(\begin{array}{cc}
\rho_{11}^{n} & \rho_{12}^{n} \\
\rho_{12}^{n} & \rho_{22}^{n}
\end{array}\right) .
$$


Далее, предположим, что секторам $S_{0}^{-\theta_{1}}, \ldots, S_{-\theta_{n-1}}^{-\pi}$ поставлены в соответствие матрицъ

$$
\left(\begin{array}{cc}
\rho_{11}^{1} & -\rho_{12}^{1} \\
-\rho_{12}^{1} & \rho_{22}^{1}
\end{array}\right), \quad \ldots, \quad\left(\begin{array}{cc}
\rho_{11}^{n} & -\rho_{12}^{n} \\
-\rho_{12}^{n} & \rho_{22}^{n}
\end{array}\right) .
$$

Обозначим через $\mathscr{A}_{\lambda}$ оператор, соответствующий матрицам (4.6), (4.7) при указанном разбиении пространства $\mathbb{R}^{2}$ на секторы. Далее, пусть $\mathscr{A}_{\lambda}^{D} u \mathscr{A}_{\lambda}^{N}-$ операторы, задаваемые матрицами (4.6) в секторах полупространства $\{(x, y)$ : y $>0\}$ и граничными условиями Дирихле и Неймана соответственно. Тогда для любого $\lambda$, такого, что $\operatorname{Re} \lambda>0$, ядро оператора $\mathscr{A}_{\lambda}$ тривиально при условии, что для этого же $\lambda$ тривиальны ядра операторов $\mathscr{A}_{\lambda}^{D} u \mathscr{A}_{\lambda}^{N}$.

Доказательство. Доказательство для случая двух секторов в полупространстве дано в [9, лемма 22]. С необходимыми изменениями оно переносится на случай многих секторов.

\section{§5. Результат об оптимальной регулярности}

Теперь можно сформулировать наш основной результат об оптимальной регулярности эллиптической задачи вблизи гетерогенной вершины Неймана.

Теорема 5.1. Пусть $\Pi \subseteq \mathbb{R}^{3}$-ограниченная область, такая, что она и ее замыкание $\bar{\Pi}$ имеют одну и ту же границу, причем $\bar{\Pi}-$ полиэдральное 3 -многообразие с краем. Пусть ає - вериина многообразия $\bar{\Pi}$, и предположим, что выполнены следующие условия.

1. Коэффичиентная функиия $\mu$ на П эллиптична и принимает значения в множестве вещественных симметрических положительно определенных матрии, размера $3 \times 3$.

2. Существует триангулячия многообразия $\bar{\Pi}$ (конечнъмм) евклидовым комплексом $K$, таким, что н постоянна на внутренности каждой 3-клетки, принадлежсащей $K$.

3. Каждое ребро в $K$, лежсащее на гранище области П, для которого вершина аџ является одним из кониов, представляет собой мономатериалъное ребро или биматериальное внешнее ребро.

4. Показатель сингулярности любого внутреннего ребра с концом в а , получающегося из триангуляиии $K$, превышает $1 / 3$.

Тогда существует открытая окрестность $\mathscr{U}$ точки а $\mathrm{a}_{\mathbf{\Delta}} \in \mathbb{R}^{3}$, такая, что если положить $\Pi_{\bullet}:=\Pi \cap \mathscr{U} u \Gamma:=\partial \Pi \cap \mathscr{U}$, mo оператор

$$
-\left.\nabla \cdot \mu\right|_{\Pi_{\bullet}} \nabla: W_{\Gamma}^{1, p}\left(\Pi_{\bullet}\right) \rightarrow W_{\Gamma}^{-1, p}\left(\Pi_{\bullet}\right)
$$

будет топологическим изоморфизмом для некоторого $p>3$.

Замечание 5.2. 1. Условие 2 в теореме 5.1 ни в коем случае не означает, что коэффициентная функция $\mu$ обязана принимать разные значения на разных клетках.

2. Для случая оператора Лапласа (т. е. $\mu \equiv 1_{\mathbb{R}^{3}}$ ) теорема 5.1 утверждает, что одних лишь геометрических условий на П достаточно, чтобы гарантировать, что (5.1) - изоморфизм, вне зависимости от того, насколько «дикой» в действительности является локальная геометрия области П (ср. [6]). Итак, здесь мы находимся в той же ситуации, что и в случае задачи Дирихле (ср. предложения 4.6 и 4.9, а также [7, теорема 2.1]). 
K сожалению, для внутренних ребер представляется чрезвычайно трудным выяснить, тривиальны ли ядра операторов $\mathscr{A}_{\lambda}$ в общем случае (см. подробное обсуждение в [7]). Однако имеется несколько важных конфигураций, для которых известно, что это предположение выполнено.

Определение 5.3. Пусть заданы углы $-\pi=\theta_{0}<\theta_{1}<\cdots<\theta_{n}=\pi$, и пусть матрица $\hat{\mu}$ постоянна в каждом секторе $S_{j}:=S_{\theta_{j-1}}^{\theta_{j}}, j=1, \ldots, n$, в $\mathbb{R}^{2}$. Тогда будем говорить, что $\hat{\mu}$ распределена квазимонотонно, если существуют индексы $j_{\min }, j_{\max } \in\{1, \ldots, n\}$, такие, что

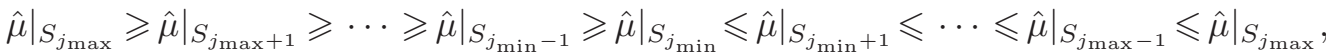
а также точка $\mathrm{x} \in \mathbb{R}^{2}$, такая, что $\mathrm{x} \in S_{j_{\max }}$ и $-\mathrm{x} \in S_{j_{\min }}$. Здесь индексы рассматриваются по модулю $n$, т. е. $S_{n+1}$ есть снова $S_{1}$.

Пример 5.4. Условие 4 теоремы 5.1 выполнено в каждом из следующих случаев.

1. Существует в точности одна плоскость, содержащая аџ и разбивающая П в окрестности точки аґ на две части, такие, что коэффициентная функция локально постоянна в каждой из этих частей. Разумеется, при этом вблизи точки ац нет внутренних ребер.

2. Существуют в точности две плоскости, содержащие точку аџ и пересекающие П, такие, что коэффициентная функция постоянна и скалярнозначна в каждой из соответствующих четырех четвертей пространства. Более того, эта последовательность скаляров монотонно возрастает, если начать с наименьшего из них и затем переходить из сектора в сектор (см. [26, теорема 6.4]).

3. Матрицы распределены квазимонотонно (ср. [7, лемма 2.1], а также [14]). $\mathrm{B}$ частности, это так, если $\mu$ скалярнозначна, а $\mathbb{R}^{2}$ разбивается на три сектора, угол раствора каждого из которых меньше $\pi$.

Замечание 5.5. Условие 3 в теореме 5.1, в частности, запрещает сочетание матриц $M_{+}, M_{-}, M_{+}$в трех соседних секторах, несмотря на то, что в нем участвуют только два разных материала.

\section{§6. Доказательство теоремы 5.1}

6.1. Локализация и отражение. Поскольку наша цель - доказать оптимальную эллиптическую регулярность в окрестности вершины а полиэдральной области П, следующая задача состоит в том, чтобы определить подходящую окрестность $\mathscr{U}$ рассматриваемой точки а, в которой мы и будем исследовать регулярность решения.

Определение 6.1. Начиная с этого момента, мы все время предполагаем, что а $\mathbf{\Delta}$ - вершина полиэдральной области П и что $\varphi_{\mathrm{a}_{\mathbf{\Delta}}}$ выбрана, как в теореме 3.10. Кроме того, определим евклидов комплекс $K_{\mathscr{C}}^{+}$как комплекс $L_{\overline{\mathscr{C}}} \cap \overline{\mathscr{C}_{+}}$.

Пусть теперь заданы срезающая функция $\eta \in C_{0}^{\infty}\left(\mathbb{R}^{d}\right)$ с носителем $\operatorname{supp}(\eta)$ $\subseteq \mathscr{U}$ и правая часть $f \in\left(W^{1, p^{\prime}}(\Pi)\right)^{\prime}$ для некоторого $\left.\left.p \in\right] 3,6\right]$. В следующей лемме выводится уравнение для усеченной функции $\eta v$.

Лемма 6.2 [10, гл. 5.1]. Пусть $p \in] 3,6]$, и пусть $\mathscr{U} \subseteq \mathbb{R}^{3}$-открытое множество, такое, что область П॰ $:=\Pi \cap \mathscr{U}$ снова липиищева. Положим 
$\Gamma:=\partial \Pi \cap \mathscr{U} . \Pi$ Путь $v \in W^{1,2}(\Pi)-($ единственное $)$ решение уравнения

$$
-\nabla \cdot \mu \nabla v+v=f \in\left(W^{1, p^{\prime}}(\Pi)\right)^{\prime} .
$$

Тогда $\eta v \in W_{\Gamma}^{1,2}\left(\Pi_{\bullet}\right) u$

$$
-\left.\nabla \cdot \mu\right|_{\Pi_{\bullet}} \nabla(\eta v)=g \in W_{\Gamma}^{-1, p}\left(\Pi_{\bullet}\right),
$$

где $g$ непрерьвно зависит от $f$.

Эта лемма утверждает, что для заданных $f \in\left(W^{1, p^{\prime}}(\Pi)\right)^{\prime}$ и вариационного решения $v$ задачи (6.1) функция $\eta v$ является вариационным решением задачи (6.2) со смешанными граничными условиями (в том смысле, что запись $g \in L^{2}\left(\Pi_{\bullet}\right)$ подразумевает условие Неймана на Г и условие Дирихле на $\left.\partial \bullet_{\bullet} \backslash \Gamma\right)$. Таким образом, можно перефразировать исходную задачу о регулярности поведения решения в окрестности неймановского ребра в виде вопроса об оптимальной регулярности для смешанной граничной задачи.

На следующем шаге (с этого места полагая $\mathscr{U}:=\varphi_{\text {ал }}(\mathscr{C})$ ) мы преобразуем нашу задачу с помощью кусочно линейного гомеоморфизма $\phi=\varphi_{\text {а }}^{-1}$, построенного в теореме 3.10. Как указано в начале $§ 3$, уравнение (6.2) преобразуется тогда в

$$
-\nabla \cdot \underline{\mu} \nabla u=h \in W_{\Sigma}^{-1, p}\left(\mathscr{C}_{+}\right),
$$

где $u:=(\eta v) \circ \phi^{-1}$. В дополнение к этому мы знаем, что регулярность поведения решений одинакова для конфигураций, связанных лишь билипшицевыми преобразованиями (ср. предложение 3.1). Поэтому решающим шагом в доказательстве основного результата теоремы 5.1 является следующая лемма.

Лемма 6.3. Пусть $\Pi \subseteq \mathbb{R}^{3}$, аџ $\in \partial \Pi$ и $\mu$ такие же, как в теореме 5.1, a $\varphi_{\mathrm{a}_{\mathbf{\Delta}}}-$ такое же, как в теореме 3.10. Положим $\Pi_{\bullet}:=\mathscr{U} \cap \Pi=\varphi_{\mathrm{a}_{\mathbf{\Delta}}}(\mathscr{C}) \cap \Pi$ u определим $\underline{\mu}$ как коэффициентную функиию, полученную на $\mathscr{C}_{+}$uз $\left.\mu\right|_{\Pi} c$ помощъю билипиицева преобразования $\varphi_{\mathrm{a}}^{-1}$ (см. предложсение 3.1$)$.

Тогда отображение $-\nabla \cdot \underline{\mu} \nabla: W_{\Sigma}^{1, p}\left(\mathscr{C}_{+}\right) \rightarrow W_{\Sigma}^{-1, p}\left(\mathscr{C}_{+}\right)$является топологическим изоморфизмом для некоторого $р>3$.

Это снова задача со смешанными граничными условиями, но теперь неймановская часть границы $\Sigma$ плоская. Поэтому можно применить стандартное рассуждение, основанное на отражении (см. [9, предложение 17]). В результате приходим к задаче Дирихле на кубе $\mathscr{C}$, которая будет рассмотрена ниже. Итак, чтобы доказать лемму 6.3, достаточно показать, что справедливо следующее утверждение.

\section{Лемма 6.4. Отображсение}

$$
-\nabla \cdot \hat{\mu} \nabla: W_{0}^{1, p}(\mathscr{C}) \rightarrow W^{-1, p}(\mathscr{C})
$$

где коэффиииентная функиия $\hat{\mu}$ получена из $\underline{\mu}$ четным отражением, является топологическим изоморфизмом для некоторого $р>3$.

Очевидно, отображение (6.4) определяет задачу Дирихле на полиэдральной области. Чтобы найти триангуляцию куба $\overline{\mathscr{C}}$, на 3 -клетках которой коэффициентная функция $\hat{\mu}$ постоянна, введем следующее определение. 
Определение 6.5. Определим комплекс $K_{\overline{\mathscr{C}}}$ как объединение всех клеток комплекса $K_{\mathscr{C}}^{+}$и всех клеток, являющихся образами клеток комплекса $K_{\mathscr{C}}^{+}$при отображении $(x, y, z) \mapsto(x,-y, z)$.

Замечание 6.6. Триангуляция $K_{\overline{\mathscr{C}}}$ куба $\overline{\mathscr{C}}$, вообще говоря, отлична от $L_{\overline{\mathscr{C}}}$, но совпадает с ней на $\overline{\mathscr{C}}+$. Кроме того, коэффициентная функция $\hat{\mu}$ постоянна на внутренности каждой 3 -клетки триангуляции $K_{\overline{\mathscr{C}}}$. Таким образом, этот комплекс определяет ребра, существенные для задачи Дирихле в лемме 6.4. При этом важно, что вся конфигурация коэффициентов на $\mathscr{C}$ связана с исходной конфигурацией коэффициентов в П таким образом, который в дальнейшем позволит нам проконтролировать эллиптические реберные особенности с помощью условий, наложенных на коэффициентную функцию $\mu$ в П.

В соответствии с предложением 4.6 для доказательства леммы 6.4 достаточно оценить реберные особенности. Подробности изложены в следующих двух разделах.

6.2. Поиск ребер. Чтобы найти все эти ребра, для заданной вершины ац, рассмотрим коэффициентную функцию $\hat{\mu}$ из леммы 6.4. Вспомним в этом контексте замечание 6.6 , а также кусочно линейное отображение $\varphi_{\mathrm{a}_{\star}}$ и комплекс $K_{\overline{\mathscr{C}}}$ из определений 6.1 и 6.5 .

Определение 6.7. Для любых $t_{0}>0$ и векторов a $\in \mathbb{R}^{3}$ и $\mathrm{b} \in \mathbb{R}^{3} \backslash\{0\}$ назовем множество $\{\mathrm{a}+t \mathrm{~b}, t \in] 0, t_{0}[\}$ отрезком с начальной точкой а. Отрезок в П назовем мономатериальным, если он содержится во внутренности одной из 3 -клеток комплекса $K_{\Pi}$. Отрезок в П назовем биклеточным, если он лежит во внутренности некоторой 2-грани комплекса $K_{\Pi}$.

Замечание 6.8. Напомним, что матрица коэффициентов $\mu$ не обязана принимать разные значения на двух 3-клетках, примыкающих к 2-грани, содержащей биклеточный отрезок.

В дальнейшем существенны следующие факты о ребрах из $K_{\overline{\mathscr{C}}}$.

Лемма 6.9. Для ребра Е через $\stackrel{\circ}{E}$ обозначим его относительную внутренность.

1. Любое ребро комплекса $K_{\overline{\mathscr{C}}}$, лежащее в $\partial \mathscr{C}$, является либо мономатериальным ребром, либо биматериальным внешним ребром.

2. Один из концов любого ребра $E$ комплекса $K_{\bar{C}}$, пересекающего $\mathscr{C}_{+}$, находится в точке 0. Следовательно, отрезок $\varphi_{\mathrm{a}_{\mathbf{\Delta}}}(\stackrel{\circ}{E})$ начинается в точке а. Таким образом, $\varphi_{\mathrm{a}_{\mathbf{\Delta}}}(\stackrel{\circ}{E})$ - либо мономатериальный или биклеточный отрезок в П, либо содержится в ребре комплекса $K_{\Pi}$, т.е. в исходной триангуляиии множества $\bar{\Pi}$.

3. Для любого ребра $E \subseteq \Sigma$ множество $\varphi_{\mathrm{a}_{\mathbf{\Delta}}}(\stackrel{\circ}{E}) \subseteq \partial \Pi$ является отрезком $с$ начальной точкой аџ и потому либо мономатериальным отрезком, либо частъю мономатериального ребра, либо частью биматериального внешнего ребра, уже присутствовавшего в исходной триангуляиии $K_{\Pi}$.

Доказательство. Все три утверждения следуют из определения триангуляции полиэдра $\varepsilon \overline{\mathscr{C}}$, индуцированной комплексом $h\left(L_{\text {a }}^{\star}\right)$ (см. доказательство теоремы 3.10). 
1. Поскольку ребра из $\partial \mathscr{C}_{-} \backslash \bar{\Sigma}$ являются отражениями ребер из $\partial \mathscr{C}_{+} \backslash \bar{\Sigma}$, достаточно доказать утверждение для ребер, содержащихся в последнем множестве, и для ребер из $\partial \Sigma$. Рассматривая ребра из $\partial \mathscr{C}_{+} \backslash \bar{\Sigma}$, важно помнить, что значение $\varepsilon$ было выбрано так, чтобы гарантировать, что линк комплекса $h\left(L_{\text {a }}^{\star}\right)$ не пересекается с $\varepsilon \overline{\mathscr{C}}$. Таким образом, любое ребро, содержащееся в $\partial \mathscr{C}_{+} \backslash \bar{\Sigma}$, либо является «естественным» ребром границы $\partial \mathscr{C}$, либо получается из пересечения какой-либо 2 -клетки из $h\left(L_{\mathrm{a}}^{\star}\right)$, одна из вершин которой есть точка 0, с $\partial(\varepsilon \mathscr{C})$. Поскольку такая 2-клетка примыкает в точности к двум тетраэдрам, получающееся ребро может быть лишь мономатериальным или биматериальным. Наконец, каждое ребро $E$ из $\partial \Sigma$ по построению является ребром в точности одного элемента из $K_{\mathscr{C}}^{+}$(см. определение 6.1). Вспоминая определение 6.5 комплекса $K_{\overline{\mathscr{C}}}$, понимаем, что $E-$ мономатериальное или биматериальное ребро в $K_{\overline{\mathscr{C}}}$, в зависимости от того, отличается или нет коэффициентная функция на отраженной ячейке от коэффициентной функции на соответствующей ячейке в $K_{\mathscr{C}}^{+}$.

2. По построению все ребра, пересекающие внутренность полиэдра $\left|h\left(L_{a}^{\star}\right)\right|$, начинаются в нуле. Ясно, что это свойство не пропадает при пересечении с $\varepsilon \overline{\mathscr{C}}$. Поэтому если $E$ пересекается с $\mathscr{C}_{+}$, то $\varphi_{\mathrm{a}_{\mathbf{\Delta}}}(\stackrel{\circ}{E})$ должно быть отрезком с одним из концов в точке а৯. Но каждый такой отрезок может быть только мономатериальным или биклеточным либо должен быть частью ребра исходной триан-

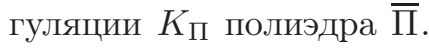

3. Каждое ребро из $\Sigma$ уже присутствует в $h\left(L_{a}^{\star}\right)$ и потому должно начинаться в точке $0 \in \mathbb{R}^{3}$. Таким образом, нужное утверждение следует из п. (5) теоремы 3.10 .

6.3. Оценки реберных особенностей. В этом разделе мы завершаем доказательство теоремы 5.1. В силу рассуждений из $\$ 4$ все в порядке, если для всех ребер триангуляции $K_{\overline{\mathscr{C}}}$ куба $\overline{\mathscr{C}}$ индуцированные операторы $\mathscr{A}_{\lambda}$ имеют тривиальное ядро для всех $\lambda$, таких, что $\operatorname{Re} \lambda \in] 0,1 / 3]$ (см. также замечание 4.7). Нужно рассмотреть следующие ребра:
(I) ребра из $\partial \mathscr{C}$,
(II) ребра из $\mathscr{C}_{+}$,
(III) ребра из $\Sigma$,
(IV) ребра из $\mathscr{C}_{-}$.

Нетрудно видеть, что ребра из п. (IV) можно рассматривать аналогично ребрам из п. (II). Итак, далее обсудим случаи (I)-(III).

(I) Ребра из $\partial \mathscr{C}$. В соответствии с п. 1 леммы 6.9 любое ребро, содержащееся в $\partial \mathscr{C}$, является мономатериальным ребром или биматериальным внешним ребром. Поэтому соответствующие операторы $\mathscr{A}_{\lambda}$ имеют при $\left.\left.\operatorname{Re} \lambda \in\right] 0,1 / 2\right]$ тривиальное ядро в силу предложений 4.9 и 4.10.

(II) Ребра из $\mathscr{C}_{+}$. Пусть $E$ - ребро из $\mathscr{C}_{+}$. Тогда $E-$ внутреннее ребро с одним из концов в $0 \in \mathbb{R}^{3}$, ср. п. 2 леммы 6.9. Рассмотрим евклидов комплекс $K_{E} \subseteq K_{\mathscr{C}}^{+}$, содержащий в точности те тетраэдры $\sigma \in K_{\mathscr{C}}^{+}$, для которых $E \subseteq \sigma$. Соответствующий двумерный сектор совпадает в этом случае со всем пространством $\mathbb{R}^{2}$. Совершим теперь обратное преобразование к исходной конфигурации в П, т. е. применим кусочно линейный гомеоморфизм $\phi:=\varphi_{\mathrm{a}_{\star}}$, ассоциированный с ац по теореме 3.10. Соответственно $E$ перейдет тогда либо в мономатериальный или биклеточный отрезок в П (каждый из которых далее рассматривается как ребро в весьма специфической конфигурации коэффициентов), либо 
в открытую часть какого-либо ребра в $K_{П}$, т. е. в исходной триангуляции полиэдра $\bar{\Pi}$ (см. лемму 6.9). В первых двух случаях реберных особенностей нет, т. е. $\operatorname{ker} \mathscr{A}_{\lambda}=\{0\}$ при $\left.\operatorname{Re} \lambda \in\right] 0,1[$ (ср. [7, гл. 5.1]), в то время как в третьем случае по предположению имеем $\operatorname{ker} \mathscr{A}_{\lambda}=\{0\}$, если $\operatorname{Re} \lambda \leqslant 1 / 3+\epsilon$. Теперь можно просто применить теорему 4.15.

(III) Ребра из $\Sigma$. Очевидно, что каждое ребро из $\Sigma$ является внутренним ребром по отношению к $\mathscr{C}$, и в силу п. 2 леммы 6.9 один из его концов есть точка $0 \in \mathbb{R}^{3}$. Если для заданного ребра $E$ из $\Sigma$ матрицы коэффициентов, отвечающие соответствующим секторам в полупространстве $\left\{(x, y, z) \in \mathbb{R}^{3}: y>0\right\}$, имеют вид

$$
M^{1}=\left(\begin{array}{lll}
m_{11}^{1} & m_{12}^{1} & m_{13}^{1} \\
m_{12}^{1} & m_{22}^{1} & m_{23}^{1} \\
m_{13}^{1} & m_{23}^{1} & m_{33}^{1}
\end{array}\right), \quad \ldots, \quad M^{n}=\left(\begin{array}{ccc}
m_{11}^{n} & m_{12}^{n} & m_{13}^{n} \\
m_{12}^{n} & m_{22}^{n} & m_{23}^{n} \\
m_{13}^{n} & m_{23}^{n} & m_{33}^{n}
\end{array}\right),
$$

то соответствующие матрицы в отраженных секторах имеют вид

$$
M_{-}^{1}=\left(\begin{array}{ccc}
m_{11}^{1} & -m_{12}^{1} & m_{13}^{1} \\
-m_{12}^{1} & m_{22}^{1} & -m_{23}^{1} \\
m_{13}^{1} & -m_{23}^{1} & m_{33}^{1}
\end{array}\right), \quad \ldots, \quad M_{-}^{n}=\left(\begin{array}{ccc}
m_{11}^{n} & -m_{12}^{n} & m_{13}^{n} \\
-m_{12}^{n} & m_{22}^{n} & -m_{23}^{n} \\
m_{13}^{n} & -m_{23}^{n} & m_{33}^{n}
\end{array}\right)
$$

(см. [9, предложение 17]). В соответствии с предложением 4.6 нужно выполнить поворот в плоскости $(x, z)$, переводящий рассматриваемое ребро в подмножество оси $z$. Это означает, что нужно рассмотреть матрицы

$$
\left(\begin{array}{ccc}
\cos \alpha & 0 & -\sin \alpha \\
0 & 1 & 0 \\
\sin \alpha & 0 & \cos \alpha
\end{array}\right) M\left(\begin{array}{ccc}
\cos \alpha & 0 & \sin \alpha \\
0 & 1 & 0 \\
-\sin \alpha & 0 & \cos \alpha
\end{array}\right)
$$

где в качестве $M$ последовательно берутся $M^{1}, \ldots, M^{n}, M_{-}^{1}, \ldots, M_{-}^{n}$, а $\alpha-$ угол между соответствующим ребром и осью $z$. Непосредственное вычисление показывает, что получающийся блок размера $2 \times 2$ имеет вид

$$
\left(\begin{array}{cc}
\rho_{11}^{1} & \rho_{12}^{1} \\
\rho_{12}^{1} & \rho_{22}^{1}
\end{array}\right), \quad \ldots, \quad\left(\begin{array}{cc}
\rho_{11}^{n} & \rho_{12}^{n} \\
\rho_{12}^{n} & \rho_{22}^{n}
\end{array}\right)
$$

в секторах из полупространства $\left\{(x, y, z) \in \mathbb{R}^{3}: y>0\right\}$ и

$$
\left(\begin{array}{cc}
\rho_{11}^{1} & -\rho_{12}^{1} \\
-\rho_{12}^{1} & \rho_{22}^{1}
\end{array}\right), \quad \ldots, \quad\left(\begin{array}{cc}
\rho_{11}^{n} & -\rho_{12}^{n} \\
-\rho_{12}^{n} & \rho_{22}^{n}
\end{array}\right)
$$

в отраженных секторах из полупространства $\left\{(x, y, z) \in \mathbb{R}^{3}: y<0\right\}$. В этой конфигурации можно применить лемму 4.16, чтобы свести задачу, заданную на секториальном разбиении всего пространства $\mathbb{R}^{2}, \mathrm{k}$ задачам Дирихле и Неймана на секториальном разбиении полупространства.

Таким образом, остается доказать следующую лемму.

Лемма 6.10. Пусть $\underline{\mu}$ - преобразованная коэфбициентная функиия на $\mathscr{C}_{+}$, и пусть $\mathscr{A}_{\lambda}^{D}$ u $\mathscr{A}_{\lambda}^{N}$-операторные пучки Штурма-Лиувилля, отвечаюшие

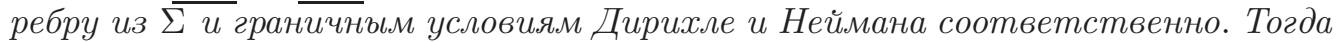
ядра операторов $\frac{\mathscr{A}_{\lambda}^{D}}{\mathscr{A}_{\lambda}^{N}}$ тривиальны при $\left.\left.\operatorname{Re} \lambda \in\right] 0,1 / 2\right]$.

Доказательство. Пусть $E$ - ребро из $\Sigma$. Рассмотрим на этот раз евклидов комплекс $K_{E}$, содержащий в точности те тетраэдры $\sigma \in K_{\mathscr{C}}^{+}$, для которых $E \subseteq \sigma$ 
(ср. с определением 6.1). Проекция полиэдра $\left|K_{E}\right|$ на любую перпендикулярную к $E$ плоскость локально вблизи $E$ является сектором, а именно, соответствующим полупространством в $\mathbb{R}^{2}$. Это гарантирует выполнение предположения 2 в теореме 4.15 .

Преобразуем теперь соответствующее ребро обратно в исходную конфигурацию в П, т. е. применим отображение $\phi:=\varphi_{\mathrm{a}}$ в смысле $\S 3$. Образ ребра $E$ при этом отображении обязательно либо содержится в плоской грани одной из клеток комплекса $K_{\Pi}$, либо является частью мономатериального ребра или биматериального внешнего ребра, уже присутствовавшего в исходной триангуляции $K_{\Pi}$ (см. п. 3 леммы 6.9). Итак, разрез через П• перпендикулярно $\phi(E)$ в окрестности образа $\phi(E)$ выглядит, как показано на рис. 2. Заметим, что в силу определения биматериального внешнего ребра оба угла раствора секторов в третьем случае не превосходят $\pi$.
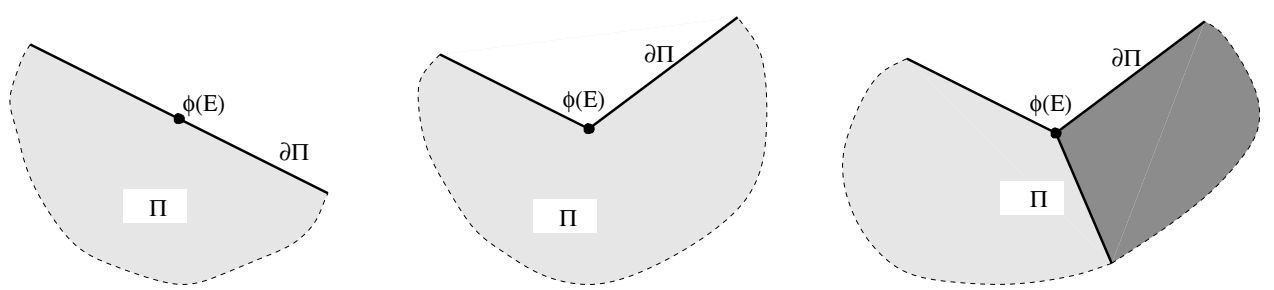

Рис. 2. Разрез через П• перпендикулярно $\phi(E)$ для мономатериального отрезка, мономатериального ребра и биматериального ребра соответственно.

Для этих конфигураций полоса $\left\{\lambda: 0<\operatorname{Re} \lambda \leqslant \frac{1}{2}\right\}$ не содержит сингулярных чисел соответствующих операторных пучков $\mathscr{A}_{\lambda}$ в силу предложений 4.9 и 4.10. Итак, применение теоремы 4.15 опять приводит к нужному утверждению.

\section{§7. Заключительные замечания}

Замечание 7.1. Мы полагаем, что наши предположения в общем случае являются также и необходимыми. Точнее, если одно из участвующих в задаче ребер допускает показатель сингулярности меньше 1/3, то [9, лемма 14] позволяет построить функцию на $\mathbb{R}^{3}$, градиент которой не лежит в $L^{3}$. Применяя подходящую срезающую функцию, можно добиться, чтобы носитель лежал в подходящем конусе, причем особенность появляется в его вершине.

Замечание 7.2. Доказанный в статье результат о регулярности легко переносится на задачи с граничными условиями Робина, поскольку соответствующий граничный оператор компактен относительно главной части.

Благодарности. Мы признательны Хансу-Гюнтеру Боте за полезные обсуждения, касающиеся разд. 3.1. Мы благодарны рецензенту, который, в частности, предложил более естественный способ обсуждения особенностей для ребер полукуба $\mathscr{C}_{+}$, чем в более ранней версии статьи. 


\section{ЛитерАТУРА}

[1] P. Alexandroff, H. Hopf, Topologie, Grundlehren der math. Wissenschaften, vol. 45, Springer-Verlag, Berlin, 1935; исправленное репринтное издание, Springer-Verlag, Berlin-Heidelberg-New York, 1974.

[2] R. H. Bing, The Geometric Topology of 3-Manifolds, AMS Colloquium Publications, vol. 40, Amer. Math. Soc., Providence, RI, 1983.

[3] M. Chipot, D. Kinderlehrer, G. V. Caffarelli, Smoothness of linear laminates, Arch. Rational Mech. Anal., 96:1 (1986), 81-96.

[4] M. Costabel, M. Dauge, S. Nicaise, Singularities of Maxwell interface problems, M2AN, Math. Model. Numer. Anal., 33:3 (1999), 627-649.

[5] A. Cianchi, V. Maz'ya, Neumann problems and isocapacitary inequalities, J. Math. Pures Appl., 89:1 (2008), 71-105.

[6] M. Dauge, Neumann and mixed problems on curvilinear polyhedra, Integral Equations Operator Theory, 15:2 (1992), 227-261.

[7] J. Elschner, H.-C. Kaiser, J. Rehberg, G. Schmidt, $W^{1, q}$ regularity results for elliptic transmission problems on heterogeneous polyhedra, Math. Models Methods Appl. Sci., $\mathbf{1 7 : 4}$ (2007), 593-615.

[8] J. Elschner, J. Rehberg, G. Schmidt, Optimal regularity for elliptic transmission problems including $C^{1}$ interfaces, Interfaces Free Bound., 9:2 (2007), 233-252.

[9] R. Haller-Dintelmann, H.-C. Kaiser, J. Rehberg, Elliptic model problems including mixed boundary conditions and material heterogeneities, J. Math. Pures Appl., 89:1 (2008), 25-48.

[10] R. Haller-Dintelmann, J. Rehberg, Maximal parabolic regularity for divergence operators including mixed boundary conditions, J. Differential Equations, 247 (2009), 1354-1396.

[11] D. Hömberg, Ch. Meyer, J. Rehberg, W. Ring, Optimal control for the thermistor problem, SIAM J. Control Optim., 48 (2010), 3449-3481.

[12] D. Jerison, C. Kenig, The inhomogeneous Dirichlet problem in Lipschitz domains, J. Funct. Anal., 130:1 (1995), 161-219.

[13] H.-C. Kaiser, J. Rehberg, Optimal elliptic regularity at the crossing of a material interface and a Neumann boundary edge, J. Math. Sci. (N.Y.), 169:2 (2010), 145-166.

[14] D. Knees, On the regularity of weak solutions of quasi-linear elliptic transmission problems on polyhedral domains, Z. Anal. Anwendungen, 23:3 (2004), 509-546.

[15] D. Leguillon, E. Sanchez-Palenzia, Computation of Singular Solutions in Elliptic Problems and Elasticity, John Wiley \& Sons, Chichester; Masson, Paris, 1987.

[16] Y. Y. Li, M. Vogelius, Gradient estimates for solutions to divergence form elliptic equations with discontinuous coefficients, Arch. Ration. Mech. Anal., 153:2 (2000), 91-151.

[17] J. Luukkainen, J. Väisälä, Elements of Lipschitz topology, Ann. Acad. Sci. Fenn., Ser. A I Math., 3:1 (1977), 85-122.

[18] В. Г. Мазья, Пространства С. Л. Соболева, Изд-во ЛГУ, Ленинград, 1985.

[19] V. Maz'ya, J. Rossmann, Elliptic Equations in Polyhedral Domains, Math. Surveys and Monographs, vol. 162, Amer. Math. Soc., Providence, RI, 2010.

[20] V. Maz'ya, J. Rossmann, Weighted $L_{p}$ estimates of solutions to boundary value problems for second order elliptic systems in polyhedral domains, Z. Angew. Math. Mech., 83:7 (2003), 435-467.

[21] V. Maz'ya, J. Elschner, J. Rehberg, G. Schmidt, Solutions for quasilinear evolution systems in $L^{p}$, Arch. Ration. Mech. Anal., 171:2 (2004), 219-262.

[22] В. Г. Мазья, С. А. Назаров, Особенности решений задачи Неймана в конической mочке, Сиб. матем. журн., 30:3 (1989), 52-63. 
[23] E. E. Moise, Geometric Topology in Dimensions 2 and 3, Graduate Texts in Math., vol. 47, Springer-Verlag, New York-Heidelberg, 1977.

[24] S. Nicaise, Polygonal interface problems, Methoden und Verfahren der Mathematischen Physik, Bd. 39, Verlag Peter D. Lang, Frankfurt/M, 1993.

[25] S. Nicaise, A.-M. Sändig, General interface problems. I, II, Math. Methods Appl. Sci., 17:6 (1994), 395-429, 431-450.

[26] M. Petzoldt, Regularity results for Laplace interface problems in two dimensions, Z. Anal. Anwendungen, 20:2 (2001), 431-455.

[27] G. Savaré, Regularity results for elliptic equations in Lipschitz domains, J. Funct. Anal., 152:1 (1998), 176-201.

[28] L. Siebenmann, D. Sullivan, On complexes that are Lipschitz manifolds, in: Geometric Topology (Proc. 1977 Georgia Topology Conf., Athens/GA 1977), Academic Press, New York-London, 1979, 503-525.

[29] И. Е. Тамм, Основы теории электричества, Физматлит, М., 1989.

[30] D. Zanger, The inhomogeneous Neumann problem in Lipschitz domains, Comm. Partial Differential Equations, 25:9-10 (2000), 1771-1808.

Technische Universität Darmstadt

e-mail: haller@mathematik.tu-darmstadt.de

Weierstrass Institute, Berlin

e-mail: Wolfgang.Hoeppner@wias-berlin.de

Weierstrass Institute, Berlin

e-mail: Hans-Christoph.Kaiser@wias-berlin.de

Weierstrass Institute, Berlin

Joachim.Rehberg@wias-berlin.de

Freie Universität Berlin

ziegler@math.fu-berlin.de
Поступило в редакцию 31 января 2012 г. 